University of Wollongong

Research Online

Australian Institute for Innovative Materials -

Papers

Australian Institute for Innovative Materials

$1-1-2017$

\title{
3D printing of tough hydrogel composites with spatially varying materials properties
}

\author{
Shannon Bakarich \\ University of Wollongong, seb798@uowmail.edu.au \\ Robert A. Gorkin III \\ University of Wollongong, rgorkin@uow.edu.au \\ Reece Gately \\ University of Wollongong, rdg604@uowmail.edu.au \\ Sina Naficy \\ University of Wollongong, snaficy@uow.edu.au \\ Marc in het Panhuis \\ University of Wollongong, panhuis@uow.edu.au
}

See next page for additional authors

Follow this and additional works at: https://ro.uow.edu.au/aiimpapers

Part of the Engineering Commons, and the Physical Sciences and Mathematics Commons

\footnotetext{
Research Online is the open access institutional repository for the University of Wollongong. For further information
} contact the UOW Library: research-pubs@uow.edu.au 


\title{
3D printing of tough hydrogel composites with spatially varying materials properties
}

\begin{abstract}
Biofabrication is the process of transforming materials into systems that reproduce biological structure and function. Previous attempts to create biomimetic systems have often used single materials shaped into limited configurations that do not mimic the heterogeneous structure and properties of many biological tissues. The identification of new bio-inspired materials alongside the development of appropriate fabrication techniques is the key to overcoming the challenge of replicating the functional gradients of these heterogeneous tissues. This paper presents a new extrusion-based gradient printing system that utilizes custom software to control the rates at which two inks are dispensed through a mixing nozzle. The printer was used to fabricate a range of composite materials containing varying blends of a tough alginate/poly(acrylamide) ionic covalent entanglement hydrogel and an acrylated urethane based UV-curable adhesive material. The hard adhesive material acted as particulate reinforcement within the matrix of composites printed with a large hydrogel volume fraction. The composite materials were characterized mechanically and their performance could be modeled with standard composite theory. The platform of a 3D printer allowed these composite materials to be fabricated directly with a smooth and continuous gradient of modulus between the soft hydrogel and harder acrylated urethane material, which may be useful in the development of bio-inspired structures such as artificial tendons.

\section{Disciplines}

Engineering | Physical Sciences and Mathematics

\section{Publication Details}

Bakarich, S. E., Gorkin III, R., Gately, R., Naficy, S., in het Panhuis, P. \& Spinks, G. M. (2017). 3D printing of tough hydrogel composites with spatially varying materials properties. Additive Manufacturing, 14 24-30.
\end{abstract}

\section{Authors}

Shannon Bakarich, Robert A. Gorkin III, Reece Gately, Sina Naficy, Marc in het Panhuis, and Geoffrey M. Spinks 


\section{D printing of tough hydrogel composites with spatially varying materials properties}

Shannon E. Bakarich ${ }^{1}$, Robert Gorkin III ${ }^{1}$, Reece Gately ${ }^{2}$, Sina Naficy ${ }^{1,3}$, Marc in het Panhuis ${ }^{1,2}$ and Geoffrey M. Spinks ${ }^{1,3 *}$

1. Intelligent Polymer Research Institute, ARC Centre of Excellence for Electromaterials Science, AIIM Facility, University of Wollongong, Wollongong, NSW 2522, Australia

2. Soft Materials Group, School of Chemistry, University of Wollongong, Wollongong, NSW 2522, Australia

3. School of Mechanical, Materials and Mechatronic Engineering, University of Wollongong, Wollongong, NSW 2522, Australia

* E-mail: gspinks@uow.edu.au

\section{Keywords}

3D Printing, Gradient material, Tough hydrogel 


\begin{abstract}
Biofabrication is the process of transforming materials into systems that reproduce biological structure and function. Previous attempts to create biomimetic systems have often used single materials shaped into limited configurations that do not mimic the heterogeneous structure and properties of many biological tissues. The identification of new bio-inspired materials alongside the development of appropriate fabrication techniques is the key to overcoming the challenge of replicating the functional gradients of these heterogeneous tissues. This paper presents a new extrusion-based gradient printing system that utilizes custom software to control the rates at which two inks are dispensed through a mixing nozzle. The printer was used to fabricate a range of composite materials containing varying blends of a tough alginate/poly(acrylamide) ionic covalent entanglement hydrogel and an acrylated urethane based UV-curable adhesive material. The hard adhesive material acted as particulate reinforcement within the matrix of composites printed with a large hydrogel volume fraction. The composite materials were characterized mechanically and their performance could be modeled with standard composite theory. The platform of a 3D printer allowed these composite materials to be fabricated directly with a smooth and continuous gradient of modulus between the soft hydrogel and harder acrylated urethane material, such as may be useful in the development of bio-inspired structures such as artificial tendons.
\end{abstract}




\section{Introduction}

A major focus of biofabrication is creating material systems that replicate biological functions [1]. However, many bio-inspired structures are made from single materials in limited configurations and do not adequately mimic the complex heterogeneous structure and properties of biological tissue [2]. Overcoming this limitation is a challenge to identify both suitable material candidates and appropriate fabrication mechanisms amenable to create advanced structures.

Hydrogels are an attractive choice of material for mimicking soft biological tissue. Hydrogels are soft and wet materials that swell extensively in water, and have similar mechanical properties to soft tissue found in nature [3]. Despite these similarities, the use of hydrogels within clinical medicine has been limited, partly because conventional hydrogels are brittle and deficient in strength for use in structural applications [2]. Recently, there have been significant developments in new 'tough' hydrogels with fracture toughness values matching or exceeding those of natural tissue [4-7]. For instance, hydrogels have recently been described with tensile elastic modulus similar to that of skeletal muscles and fracture energy as high as $\sim 16 \mathrm{~kJ} / \mathrm{m}^{2}[8]$. These tough gels consist of waterswollen interpenetrating networks of ionically and covalently crosslinked polymers. Highly stretchable and tough hydrogels were produced when the ratio of two networks and crosslinking ratios were optimized [5].

Tough hydrogels have typically been formed using a casting process to produce a homogeneous block of material $[7,9,10]$. Yet natural tissue tends to show more complex and heterogeneous compositions [11] Tendons are a good example of heterogeneous tissue with a gradual transition or gradient of modulus throughout. The graduated stiffness allows the tendon to form a seamless interface with soft muscle at one end and hard bone at the other [12]. Creating gradients of properties within a material is a highly active area of research and several groups have attempted to fabricate synthetic functionally graded materials designed to mimic the gradient of properties within natural tissues [13]. One approach to create a gradient of mechanical properties within a hydrogel structure is casting a stack of hydrogels in a step-by-step process so that each hydrogel layer has a 
different modulus $[2,3,14]$. This method is cumbersome and produces a staggered transition rather than an optimal smooth and continuous gradient. The development of a method for fabricating tough hydrogels with a continuous gradient of material properties will allow for the production of a new generation of bio-mimetic materials to be used in bio-inspired models.

3D printing is known as a type of additive manufacturing that can construct complex 3D objects from digital models [15]. An advantage of 3D printing over other fabrication technologies, such as casting or machining, is that multiple materials can be incorporated in the construction of a single object. Composite materials and gradient structures [16-18] can be produced by digitally modelling the spatial distribution of materials within an object and selectively patterning different printing inks. Printing techniques have been developed for a range of different material types including polymers, metals, conventional hydrogels $[19,20]$ and, more recently, tough hydrogels [21-23].

Material extrusion is one of the most commonly used techniques for printing hydrogel materials [24]. Generally, this technique uses hydrogel inks housed in a syringe barrel that are extruded in the form of a fiber through the tip of the syringe. Extrusion printers have previously been used to produce composite materials by patterning fibers of different materials side-by-side [25]. Alternatively, material blends can be printed using a process where inks from two separate syringe barrels are extruded through a novel tip that mixes the two inks together before patterning them onto a substrate [26]. Coarse gradients in material properties have been produced using these printing methods by patterning different inks in incremental steps [26-28] However, no technique has yet been reported that can produce a smooth and continuous gradient in a hydrogel based material.

Herein, a modified material extrusion printing system is introduced that allows hydrogel composites to be prepared with spatially varying mechanical properties. The resulting tough hydrogel materials were characterized using mechanical testing and modelled with composite theory. A commercial acrylated urethane UV-curable adhesive (Emax $904 \mathrm{Gel}-\mathrm{SC}$ ) and alginate/poly(acrylamide) ionic 
covalent entanglement (ICE) hydrogel inks were selected to demonstrate the printing system because these two materials were recently used successfully to print fiber reinforced composites [25]. A bio-inspired structure was fabricated and characterized to demonstrate the ability of the new printing approach and hydrogel materials to mimic naturally occurring gradient structures, such as occurs in the muscle / tendon system.

\section{Materials and Methods}

\subsection{Materials}

All materials were used as-received and all solutions were prepared using Milli-Q water (resistivity = $18.2 \Omega \mathrm{cm}$ ). $\alpha$-Keto glutaric acid photo-initiator was purchased from Fluka (Australia). Acrylamide solution (40\%, for electrophoresis, sterile-filtered), alganic acid sodium salt (from brown algae with Brookfield viscosity $2 \%$ in $\mathrm{H}_{2} \mathrm{O}$ at $25{ }^{\circ} \mathrm{C}$ of $250 \mathrm{mPa}$ ), calcium chloride (minimum $93.0 \%$ granular anhydrous), ethylene glycol (rheology modifier) and N, $\mathrm{N}^{\prime}$-methylenebisacrylamide crosslinker were purchased from Sigma Aldrich (Australia). A commercial arcylated urethane UV-curable adhesive (Emax 904 Gel-SC) was purchased from Ellsworth Adhesives (Australia).

\subsection{Hydrogel Ink Formulation}

An alginate/poly(acrylamide) ionic covalent entanglement (ICE) gel ink was prepared using a previously published method [21]. The ink was prepared by dissolving $4.50 \mathrm{ml}$ of the acrylamide solution, $38.7 \mathrm{mg}$ of $\mathrm{N}, \mathrm{N}^{\prime}$-methylenebisacrylamide, $36.9 \mathrm{mg}$ of $\alpha$-keto glutaric acid, $1.5 \mathrm{ml}$ of the 0.1 $\mathrm{M}$ calcium chloride stock solution and $3.75 \mathrm{ml}$ of ethylene glycol in $5.25 \mathrm{ml}$ of Milli-Q water. A spatula was then used to dissolve $600 \mathrm{mg}$ of alganic sodium salt into the printing solution. A single drop of red food dye was added to the ink to aid in imaging the printed hydrogel.

\subsection{Material Extrusion Printing}

Ribbons of hydrogel composite materials were material extrusion printed using a custom built 3D printer. The 3D printer was built around a Sherline 8020 CNC milling stage[29] and a novel syringe controlled deposition system. The deposition system was made up of two Zaber T-LA60A-KT03 
Miniature Linear Actuators mounted to the CNC gantry by a custom designed 3D printed rig. Designs were made in Solidworks and the system was 3D printed on a Dimension uPrint Plus system (Stratasys, USA). The rig allowed for insertion of $30 \mathrm{~mL}$ or $5 \mathrm{~mL}$ standard syringe barrels preloaded with material. Plastic tubing and a Y-adaptor was used to direct the flow from the two syringes into a single stream. A disposable static chaotic mixer with 12 mixing elements (Nordson EFD) was added below the Y-adaptor to insure blending of the inks from the two syringes. A custom 3D-printed nozzle (fabricated with a Form1 3D printer, Formlabs) was attached to the bottom of the extrusion system. This nozzle was designed to extrude a flat ribbon of material having a rectangular shaped outlet with dimensions $1.5 \mathrm{~mm}$ by $15 \mathrm{~mm}$. An Opsytec Dr. Gröble $365 \mathrm{~nm}$ UV-LED smart light source with a 5W output was also integrated near the deposition system so that its illuminated area followed the print head to cure dispensed ink. Figure 1 shows the custom built printer with Figure 1 b providing a close-up view of the deposition system.

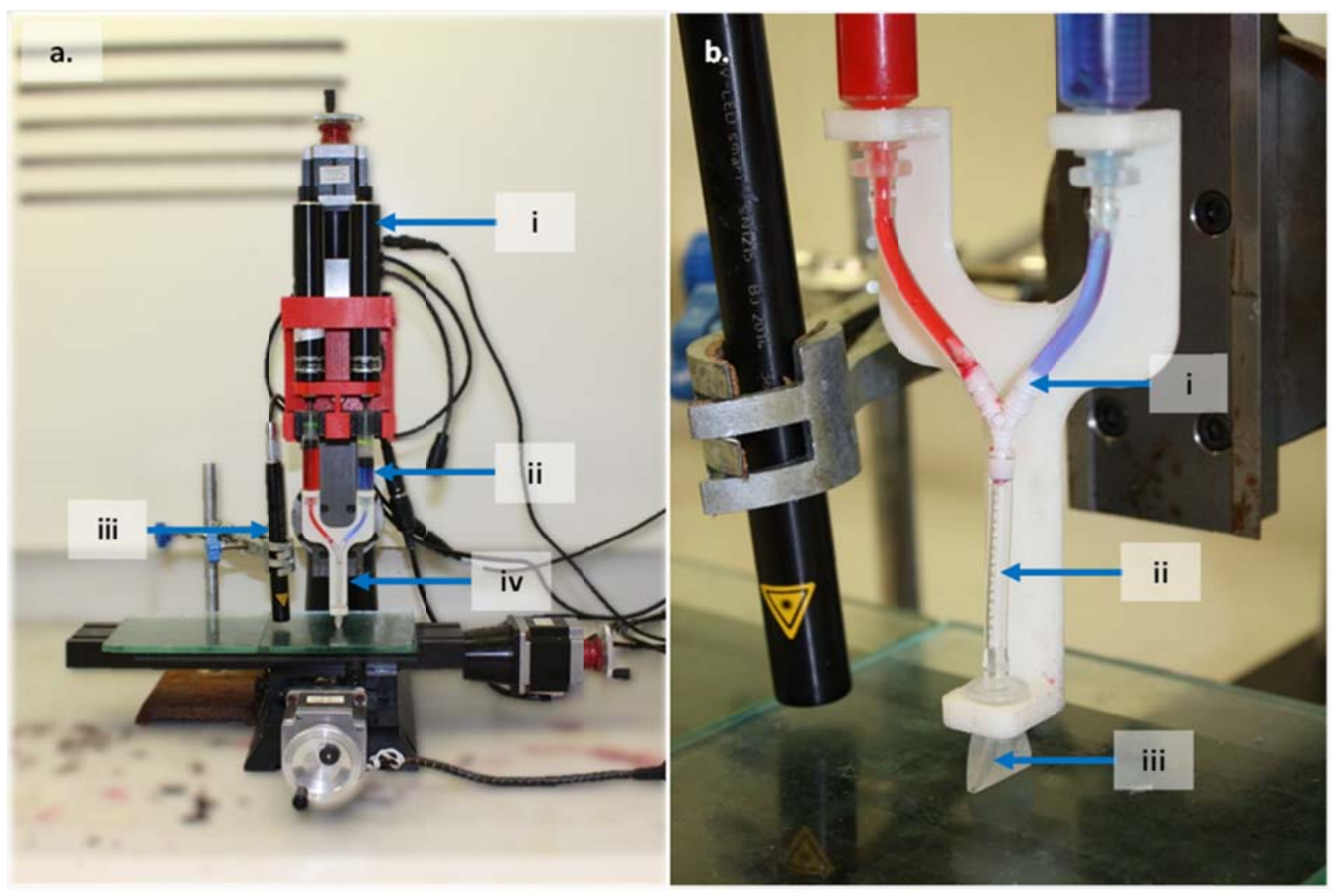


Figure 1 a. Photograph of the custom built 3D printer showing the Zaber pumps (i), syringe barrels (ii.), UV light source (iii) and the print head (iv). b. Photograph of the print head showing the position of the ' $Y$ ' adaptor (i.), the static chaotic mixer (ii.) and the custom nozzle (iii.).

To set up the print of composite ribbons the alginate/poly(acrylamide) ICE gel ink was loaded into one of the two syringes and Emax 904 Gel-SC was loaded into the other; each syringe was capped with a piston. The ribbon volume fraction and geometry was controlled using two programs running in parallel: custom software that allowed the two inks to be dispensed in different ratios, and an offthe-shelf CNC control program (LinuxCNC EMC2) controlling the movement of the print gantry. The custom program (developed in C\#, using Visual Studio 12.0) worked by adjusting the speed of the two linear actuators; which in turn delivered mechanical pressure to the pistons in the syringes and extruded material out of the $Y$ connector, through the mixer, and ultimately out of the ribbon nozzle. The net flow rate of combined material out of the nozzle had to remain constant to maintain consistency; thus the individual flow rates of the two materials from each barrel were adjusted relative to each other throughout the print. For gradient printing the flow rate of the two materials had to be linearly ramped; the speed of depression in one barrel was decreased from $100 \%$ to $0 \%$ while the other barrel experience a simultaneous speed increase from $0 \%$ to $100 \%$. The moving stage was controlled independently from the pumping actuation, and as such the flow rate and print speed were then optimized to extrude material at the appropriate speed to maintain a consistently thick layer. If the speed was too fast or too slow the print would clump or pull and show a varying thickness through the print. The optimal combined total rate of extension of the two linear actuators was set at a constant $180 \mu \mathrm{m} / \mathrm{s}$ when the milling stage was moved with a speed of $80 \mathrm{~mm} / \mathrm{min}$. The UV light using $100 \%$ intensity followed the extrusion nozzle to set the blended composite structure by partially curing the two inks.

\subsection{Post Print Processing}

A processing scheme was applied to the composite ribbons after printing to improve their mechanical properties. A Dymax BlueWave 75 Rev 2.0 UV Light Curing Spot Lamp System using a 
$19+\mathrm{W} / \mathrm{cm}^{2}$ UV source with a 1 meter light guide was used to complete the curing process. Each 30 $\mathrm{mm}$ length of ribbon was exposed to the spot of UV irradiation for $30 \mathrm{~s}$, which is the radiation dose used successfully in previous work using identical materials.[21] The composites were then immersed in $0.1 \mathrm{M}$ aqueous calcium chloride solution for $72 \mathrm{hr}$ to fully crosslink the alginate polymer network [21] and were then immersed in Milli-Q water for a further $72 \mathrm{hr}$ to reach their equilibrium swelling ratio.

\subsection{Swelling Ratio}

The swelling ratio of the printed composite samples was determined after the immersion in $0.1 \mathrm{M}$ calcium chloride and fully equilibrating in Milli-Q water. The swelling ratio here is defined as the fraction of water in the swollen network to the dry network. The swelling ratios ' $Q$ ' were calculated using:

$$
\text { Equation } 1 \quad Q=\frac{W_{S}-W_{D}}{W_{D}}
$$

where $W_{D}$ and $W_{S}$ are the dry and swollen weight of the composite, respectively. Samples were dried in an oven at $60^{\circ} \mathrm{C}$ for $72 \mathrm{hr}$.

\subsection{Imaging}

Image analysis software was used to calculate the volume fraction of the printed composite materials. Ribbons of the printed composites were imaged with a Leica 216 Macroscope. The images were loaded into MATLAB R2011a to measure the color intensity of the blue channel in images of composite printed with different ratios of the red dyed alginate/poly(acrylamide) ICE gel ink to the blue colored Emax 904 Gel-SC. The Emax 904 Gel-SC volume fraction of the printed composites was determined by comparing the intensity of the blue channel in images of the composite materials to the intensity of the blue channel in images of $0 \%$ Emax 904 Gel-SC samples (blue intensity normalized to 0) and $100 \%$ Emax 904 Gel-SC samples (blue intensity normalized to 100). The analysis was conducted on 4 separate ribbons prepared at each Emax 904 Gel-SC volume fraction. 
The images were also analyzed with Leica Application Suite V4 software to measure the diameter of Emax 904 Gel-SC particles in the composite materials.

\subsection{Mechanical Characterization}

The mechanical properties of the fully swollen printed composites were determined using a Shimadzu EZ-L Universal Mechanical Tester. Tensile specimens were prepared by cutting swollen printed composite ribbons into $30 \mathrm{~mm}$ lengths. Strips of poly(propylene) sheet were glued onto both ends of the composite tensile specimens to act as grips. The tensile specimens were attached to the mechanical tester with screw loaded clamps. Tensile tests were performed using a $500 \mathrm{~N}$ load cell and the tensile specimens were strained to failure at a rate of $10 \mathrm{~mm} / \mathrm{min}$. The applied stress was calculated using the average cross sectional area of the unstrained specimen. The failure stress and failure strain were calculated from the onset of failure. The work of extension was calculated as the area under the stress-strain curve to failure. Four repeat tests were performed on each type of composite sample with errors estimated from one standard deviation.

\section{Results and Discussion}

The aim of the present study was to develop and evaluate a custom built 3D printer equipped with a novel extrusion based deposition system to print blends of hydrogels and other materials to form gradient structures and spatially varying material properties. Emax 904 Gel-SC and alginate/poly(acrylamide) ICE hydrogel inks were identified as suitable materials to perform these tests, based on recent work where these materials were used to print fiber reinforced composites [25]. The new custom-built printer has been designed to form composites of the two extruded materials where the volume fraction of each phase could be controlled through newly developed software. The print quality has been examined using imaging techniques and the mechanical properties of the printed materials have been characterized and analyzed using standard composites theory. 


\subsection{Material Extrusion Printing}

The extrusion printer produced uniform rectangular shaped ribbons composed of a blend of the two inks. The width of the extruded rectangles was $14 \pm 1 \mathrm{~mm}$ as set by the width of the extrusion head and the ribbon thickness of $1.5 \pm 0.2 \mathrm{~mm}$ was controlled by the extrusion rate. Figure 2 a shows images of sections of the extruded ribbons printed with varying ICE hydrogel: Emax 904 Gel-SC ratios. The ratio of the two materials was varied by keeping to the total rate of extrusion constant, while varying the extrusion rate of the Emax 904 ink with respect to ICE hydrogel between $0 \%$ and $100 \%$. The appearance of the extruded samples showed a transition from red (alginate) to blue (Emax). Figure $2 \mathrm{~b}$ shows that the calculated value for the Emax $904 \mathrm{Gel}-\mathrm{SC}$ volume fraction obtained from image analysis closely agrees with the expected Emax 904 Gel-SC fraction obtained by controlling the extrusion ratio of the two inks. This result shows that the digitally controlled print heads can accurately dispense the two inks in a programed ratio. The use of stepper motors for syringe pump control provides a higher level of accuracy of the dispensed volume compared to that of the syringe pumps driven by pneumatic pressure used in previous projects.[25]
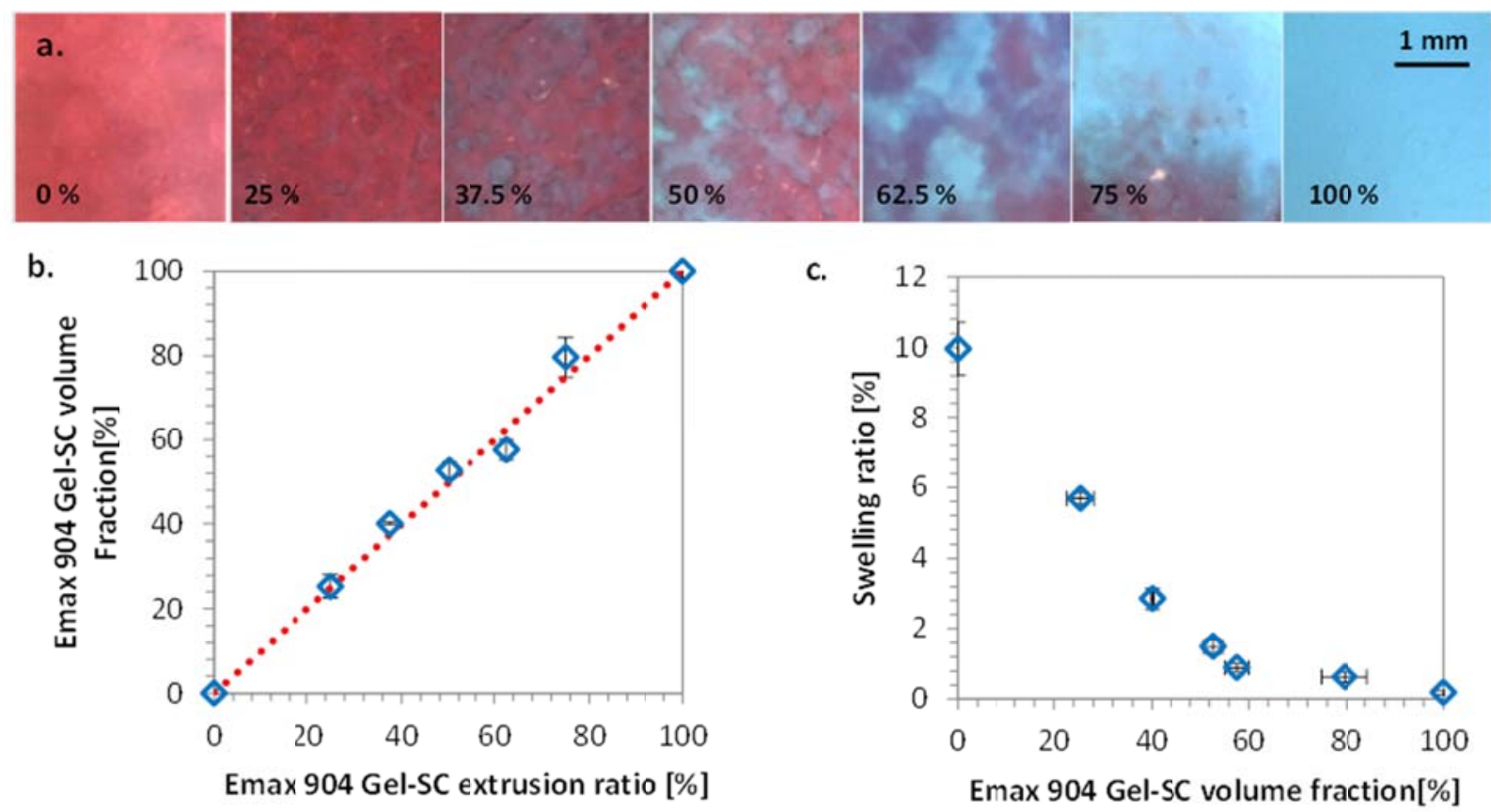

Figure 2a. Macroscope images of sample extruded ribbons printed with Emax 904 Gel-SC content of 0, 25, 37.5, 50, 62.5, 75 and $100 \%$ in blends with alginate/poly(acrylamide) ICE hydrogel. For clarity the alginate/poly(acrylamide) ICE gel is 
colored red and the Emax 904 Gel-SC is blue. b. Emax 904 Gel-SC volume fraction obtained from image analysis as a function of the software controlled Emax 904 Gel-SC extrusion ratio. c. The swelling ratio of the extruded composite materials (calculated after completion of the post printing processing) as a function of Emax 904 Gel-SC volume fraction obtained from image analysis.

The alginate/poly(acrylamide) ICE gel ink and the Emax 904 Gel-SC ink prove to be immiscible and phase separate following deposition. When the ink blend is exposed to UV irradiation the two inks begin to solidify to set the composite structure. This structure can be easily visualized as the alginate/poly(acrylamide) ICE gel ink is dyed red in color and the Emax 904 Gel-SC has a blue color. Figure 2a. includes microstructural images of the extruded ribbons printed with Emax 904 Gel-SC extrusion rates of $0,25,37.5,50,62.5,75$ and $100 \%$ of the total extrusion rate. The Emax 904 GelSC phase produces small round particles in the composite materials printed with Emax 904 Gel-SC extrusion rates of 25 and $37.5 \%$ of the total extrusion rate. The maximum diameter of the particles is $220 \pm 20 \mu \mathrm{m}$ and $510 \pm 30 \mu \mathrm{m}$, respectively, for the composite materials printed with Emax 904 Gel-SC extrusion rates of 25 and $37.5 \%$ of the total extrusion rate. A continuous Emax 904 Gel-SC phase is produced in all the composite materials printed with Emax $904 \mathrm{Gel}-\mathrm{SC}$ extrusion rates of 50 $\%$ or greater of the total extrusion rate. The alginate/poly(acrylamide) ICE gel phase does not appear to be dispersed evenly in these composites. Future modifications to the static mixer length and design could be used to modify the microstructure formed in these composites.

The extruded composite ribbons were exposed to a post printing process to complete the crosslinking of each phase[21] before undergoing mechanical characterization. A UV spot lamp was used to complete the curing process of the two inks, and then the composite ribbons were immersed in $0.1 \mathrm{M}$ calcium chloride solution to crosslink the alginate polymer network with the metal cations. All samples were then immersed in water and allowed to reach equilibrium swelling. The swelling ratio of the swollen composite materials was calculated using Equation 1. Ribbons printed entirely of the alginate/poly(acrylamide) ICE gel ink displayed a swelling ratio of $10 \pm 0.7$ and ribbons printed entirely of the Emax 904 Gel-SC ink displayed a considerably lower swelling ratio of 
just $0.20 \pm 0.04$. Figure $2 \mathrm{c}$ shows that the swelling ratio of the composite materials decreases as the volume fraction of Emax 904 Gel-SC in the composite material was increased. This result demonstrates that by varying the ratio in which the two inks are dispensed that the swelling ratio of a printed composite can be controlled over several orders of magnitude. The swelling ratio follows a linear fashion as a function of ICE hydrogel content up to the point where the Emax 904 Gel-SC ink forms the continuous phase. After this point ( $50 \%$ or greater Emax $904 \mathrm{Gel}-\mathrm{SC}$ ), the ICE phase is isolated within the continuous Emax 904 Gel-SC phase and swelling ratio deviates from the rule of mixtures.

\subsection{Mechanical Characterization}

Tensile testing was performed on segments of the printed ribbons to determine the effect of the Emax 904 Gel-SC volume fraction on the mechanical properties of the composite materials. The tensile tests were performed after the completion of the post printing process and once the composites had been equilibrated in water. The segments of the composite materials were loaded in the long direction of the ribbon. Examples of typical stress-strain curves for the printed composite ribbons are shown in Figure 3a and a summary of all data is provided in Table 1. Hydrogel ribbons printed entirely from the alginate/poly(acrylamide) ICE gel ink displayed an elastic modulus of $350 \pm$ $30 \mathrm{kPa}$, a tensile strength of $90 \pm 20 \mathrm{kPa}$, a failure strain of $38 \pm 3 \%$ and a work of extension of $19 \pm 2$ $\mathrm{kJ} / \mathrm{m}^{3}$. At the other extreme, samples printed entirely from Emax $904 \mathrm{Gel}-\mathrm{SC}$ displayed higher elastic modulus of $2300 \pm 100 \mathrm{kPa}$, tensile strength of $1100 \pm 40 \mathrm{kPa}$, failure strain of $75 \pm 7 \%$ and work of extension of $660 \pm 30 \mathrm{~kJ} / \mathrm{m}^{3}$. The reduced strength and stiffness of the alginate hydrogel can be related to its considerably higher swelling ratio compared with the Emax 904 Gel-SC [30]. As expected, the mechanical properties of the ribbons printed from a blend of the two inks varied between the two extremes. Strong adhesion between the separate phases in composite materials is essential for effective reinforcement and enhancement in matrix stiffness and strength. Previous work [25] has demonstrated that the co-crosslinking of the Emax $904 \mathrm{Gel-SC}$ and the ICE gel generated an interface strength higher than the cohesive strength of the ICE gel matrix. As a result 
of the good interface adhesion, the composite materials displayed an increase in elastic modulus, failure strength, failure strain and work of extension corresponding to an increase in Emax $904 \mathrm{Gel}-$ SC volume fraction and decrease in swelling ratio. These trends demonstrate that by varying the ratio in which these two inks are dispensed that the mechanical properties of a printed composite can be controlled over a wide range of values.
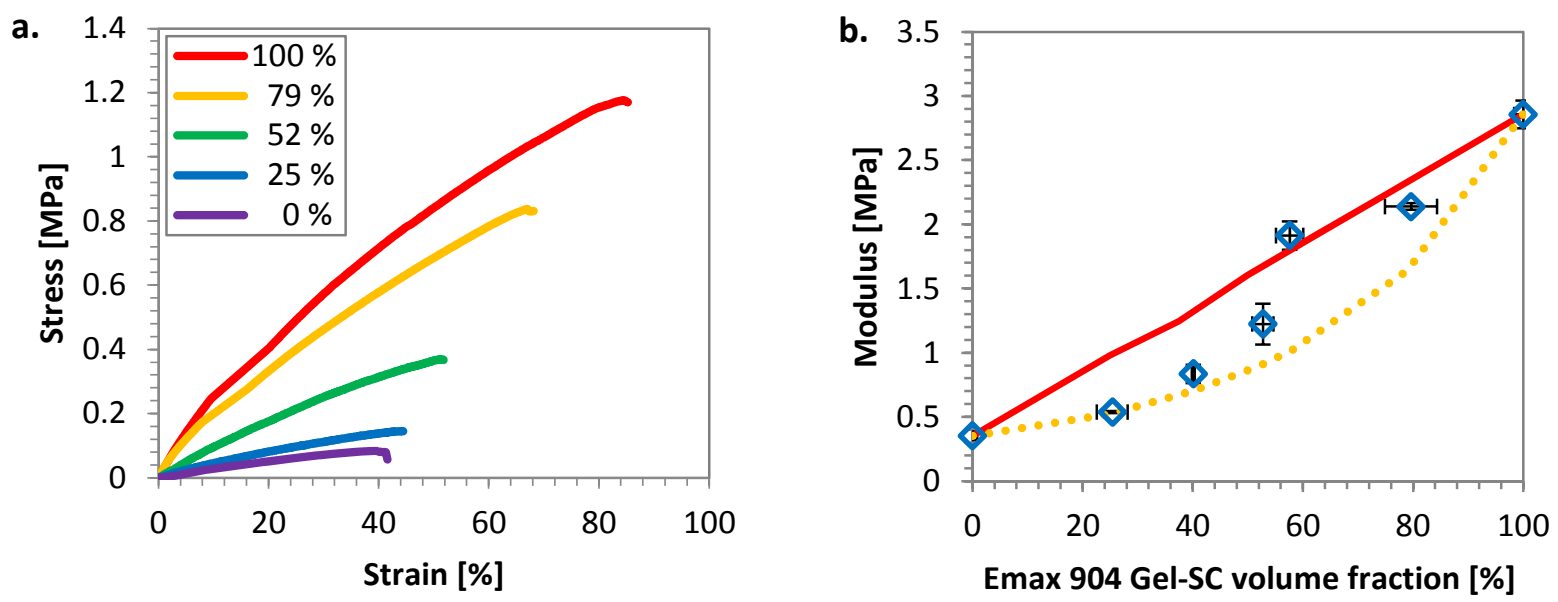

Figure 3 Typical stress-strain curves for printed composites with Emax $904 \mathrm{Gel-SC}$ volume fraction of 0, 25, 52, 79, and $100 \%$. b. Elastic modulus of the printed composites as a function of the Emax $904 \mathrm{Gel}-\mathrm{SC}$ volume fraction. Solid and dotted lines represent the theoretical upper (evaluated using Equation 2) and lower (evaluated using Equation 3) bounds for elastic modulus, respectively.

The experimental data taken from the tensile testing was compared with composite theory to determine how well the Emax 904 Gel-SC component reinforces the hydrogel matrix. The extent of agreement between the measured modulus values and the theoretical predictions depends upon the type of microstructure formed in these printed composites. At high Emax 904 Gel-SC compositions ( $58 \%$ and above), the rigid arcylated urethane phase is continuous and the composite conforms to the rule of mixtures [31] model of an ideal fiber-reinforced composite which is given by equation (2):

Equation $2 E_{\mathrm{MAX}}=E_{f} V_{f}+E_{m} V_{m}$, 
where $E_{\mathrm{MAX}}, E_{f}, E_{m}, V_{f}$ and $V_{m}$ are the upper limit for the composite modulus, elastic modulus of the filler material, elastic modulus of the matrix material and the volume fractions of the filler and the matrix, respectively. This model assumes that both phases experience identical strains under load and requires excellent adhesion between the two phases so that no slippage can occur at the interface. It has previously been shown that there is strong adhesion between the alginate/poly(acrylamide) ICE gel and Emax 904 Gel-SC when the two inks are simultaneously cured [25]. The rule of mixtures can be used to describe the elastic modulus of these fiber composites when these composite ribbons are strained in the print direction because the Emax 904 Gel-SC phase acts as continuous fibers.

The composite materials printed with Emax 904 Gel-SC volume fractions of $40 \%$ or less can be described as particulate reinforced composites of rigid Emax $904 \mathrm{Gel-SC}$ phase formed within a softer, hydrogel matrix. The reinforcing efficiency of particulate reinforcement is less than that of fiber reinforcement of the same volume fraction because the strain is not equally distributed between the two phases. Einstein was the first to develop a model to predict the elastic modulus of particulate composites [32] and in the years since a number of empirical and semi-empirical models have been proposed $[33,34]$. One such model for calculating the minimum elastic modulus $E_{M I N}$ for a particulate reinforced composite is the Ishai and Cohen equation [35] given by Equation (3):

$$
\text { Equation } 3 \quad \frac{E_{M I N}}{E_{m}}=1+\frac{V_{f}}{\delta /(\delta-1)-V_{f}^{1 / 3}}
$$

where $\delta$ is calculated as $E_{f} / E_{m}$, and $E_{M I N}$ is the lower limit for the composite modulus. This model has previously been shown to satisfactorily fit moduli data for epoxy/glass bead composites and epoxy/rubber composites[33] and also gives good agreement for the Emax 904 Gel-SC particle reinforced alginate hydrogels formed with Emax $904 \mathrm{Gel}-\mathrm{SC}$ compositions of $40 \%$ or less.

The theoretical predictions from these two composite models are shown as dashed lines in Figure 3b. It can be seen that the experimentally measured moduli values follow the lower bound 
predictions closely for Emax 904 volume fractions of 40\% and lower. At Emax 904 Gel-SC volume fractions of $58 \%$ and higher, the measured values correspond closely to the upper bound predictions. The sample prepare with a volume fraction of 52\% EMax 904 fell between the upper and lower bounds and represents a transition state between the two reinforcement processes.

Table 1. A summary of the mechanical properties of printed composite materials where $V_{E m a x}$ is the Emax 904 Gel-SC volume fraction, $Q$ is the swelling ratio, $E$ is the elastic modulus, $E_{\mathrm{MAx}}$ is the theoretical maximum elastic modulus (evaluated using Equation 2), $E_{\mathrm{MIN}}$ is the theoretical minimum elastic modulus (evaluated using Equation 3), $\sigma$ is the tensile strength, $\varepsilon$ is the failure strain and $U$ is the work of extension.

\begin{tabular}{|c|c|c|c|c|c|c|c|}
\hline $\mathrm{V}_{\mathrm{Emax}}[\%]$ & $Q$ & $\mathrm{E}[\mathrm{MPa}]$ & $\mathrm{E}_{\mathrm{MAX}}[\mathrm{MPa}]$ & $\mathrm{E}_{\mathrm{MIN}}[\mathrm{MPa}]$ & $\sigma[\mathrm{kPa}]$ & $\varepsilon[\%]$ & $\mathrm{U}\left[\mathrm{kJ} / \mathrm{m}^{3}\right]$ \\
\hline 0 & $10 \pm 0.7$ & $0.35 \pm 0.03$ & 0.35 & 0.35 & $90 \pm 20$ & $38 \pm 3$ & $19 \pm 2$ \\
\hline $25 \pm 2$ & $5.7 \pm 0.2$ & $0.54 \pm 0.01$ & 0.98 & 0.52 & $160 \pm 20$ & $42 \pm 7$ & $35 \pm 3$ \\
\hline $40 \pm 3$ & $2.9 \pm 0.3$ & $0.84 \pm 0.07$ & 1.4 & 0.70 & $270 \pm 20$ & $47 \pm 2$ & $73 \pm 9$ \\
\hline $52 \pm 2$ & $1.5 \pm 0.2$ & $1.2 \pm 0.2$ & 1.7 & 0.90 & $340 \pm 30$ & $48 \pm 7$ & $110 \pm 10$ \\
\hline $58 \pm 2$ & $0.9 \pm 0.1$ & $1.9 \pm 0.1$ & 1.8 & 1.0 & $610 \pm 30$ & $66 \pm 6$ & $140 \pm 20$ \\
\hline $79 \pm 4$ & $0.6 \pm 0.1$ & $2.1 \pm 0.1$ & 2.3 & 1.6 & $680 \pm 20$ & $67 \pm 8$ & $250 \pm 30$ \\
\hline 100 & $0.20 \pm 0.04$ & $2.9 \pm 0.1$ & 2.9 & 2.9 & $1100 \pm 40$ & $75 \pm 7$ & $660 \pm 30$ \\
\hline
\end{tabular}

\subsection{Gradient Printing}

One advantage of $3 \mathrm{D}$ printing is the ability to produce products with spatially varying material properties. In some cases a gradual transition, or gradient, in properties is desirable with many such examples seen in nature. For example, tendons are a tough band of connective tissue that connects soft muscle to hard bone. Tendons contain a gradient of mechanical properties between the two extremes of hard and soft which make a smooth interface between the muscle and bone. Here, a simplified anatomical model of a tendon-muscle-tendon system was printed to demonstrate how 
the custom built printer can be used to fabricate gradient structures. Using software control, a sample was prepared where the relative extrusion rates of the Emax 904 Gel-SC and alginate/ poly(acrylamide) inks were varied along the length of the build stage. Figure 4 a. shows the Emax $904 \mathrm{Gel}-\mathrm{SC}$ extrusion rate as a percentage of the total extrusion rate along the full $18 \mathrm{~cm}$ length of the artificial tendon. The extrusion profile was designed to produce the spatially varying structure. Figure $4 \mathrm{~b}$ includes a digital representation of the artificial tendon and Figure $4 \mathrm{c}$ includes a photograph of the printed structure. Both ends of the artificial tendon were designed to be $100 \%$ Emax 904 Gel-SC to act as rigid attachment points. Two transition zones of different length were formed adjacent to each rigid end to provide a gradient from 100\% Emax 904 Gel-SC to $100 \%$ alginate / poly(acrylamide). Transitions in color from blue to purple to red along the length of the printed structure are a visual indication of the gradient in composition and mechanical properties from hard Emax $904 \mathrm{Gel-SC}$ to the soft alginate/poly(acrylamide) ICE gel. A wedge shaped pattern is visible in the structure of the gradients in the artificial tendon which results from the laminar flow through the rectangular dye nozzle. The pattern could be removed by optimizing the flow dynamics through the nozzle. 
a.

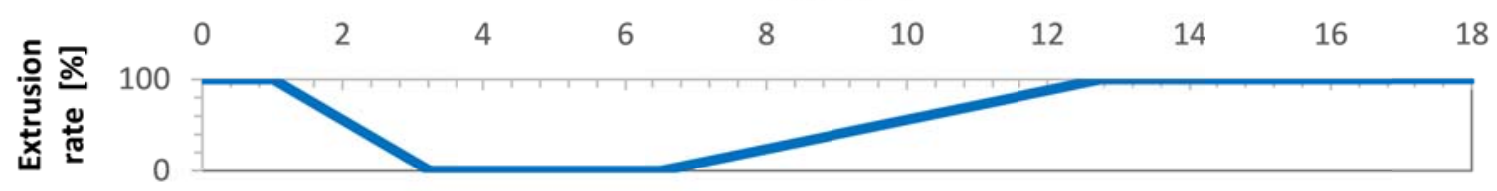

b.
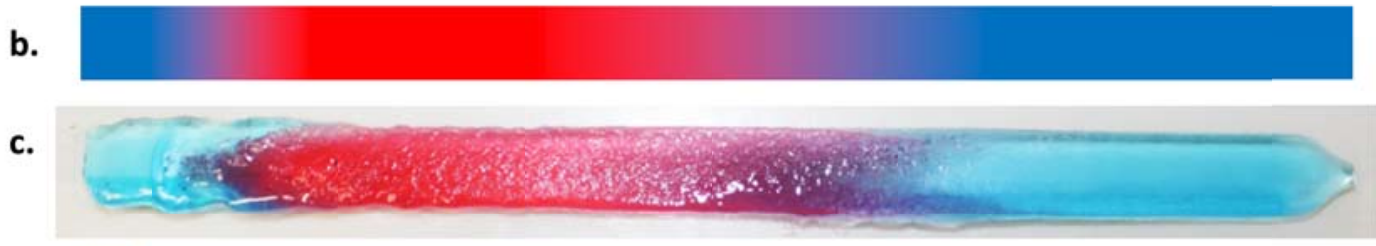

d.

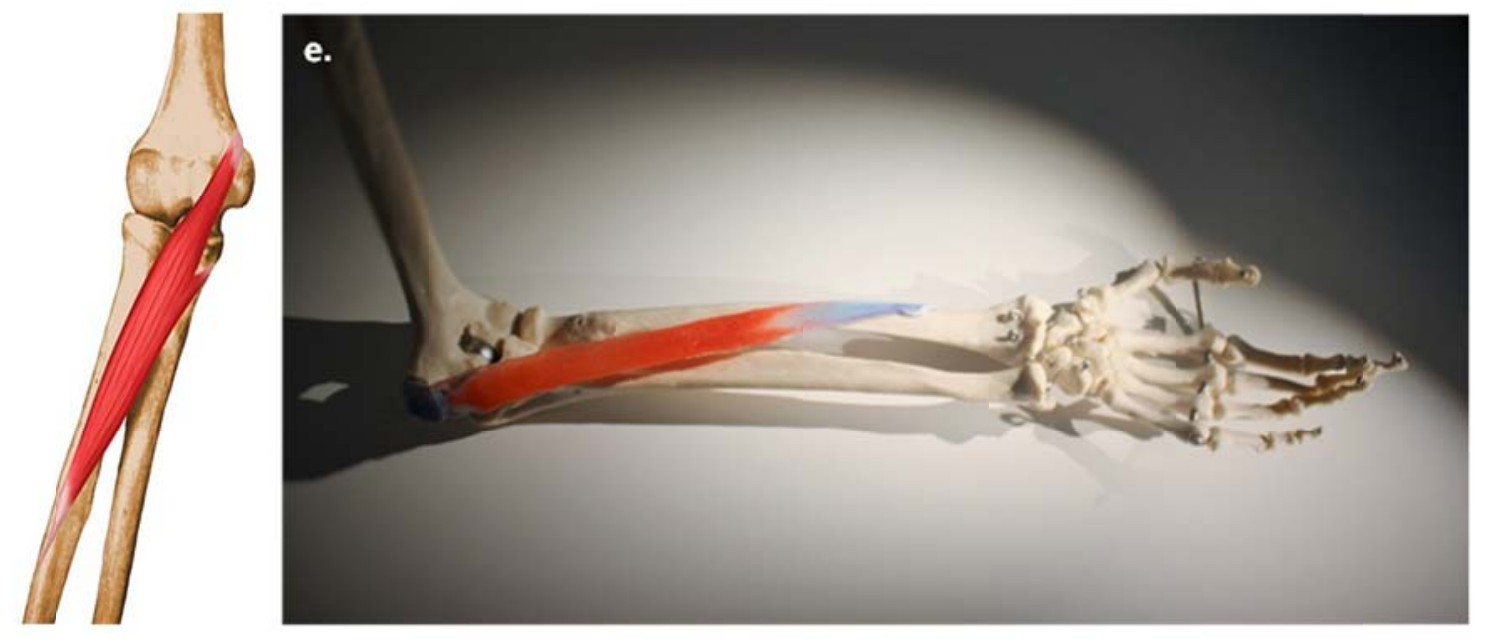

Figure 4 Demonstration of gradient printing inspired by the human tendon-muscle-tendon system in the lower arm. a. Emax $904 \mathrm{Gel}-\mathrm{SC}$ extrusion rate as a percentage of the total extrusion rate as a function of the length. b. A digital representation of a simple tendon-muscle-tendon system that shows variation in the material composition through color. The model represents gradients by the transition of blue to red. c. A photograph of a gradient gel that is a simple anatomical model of a tendon-muscle-tendon system and printed with alginate/poly(acrylamide) ICE gel and Emax 904 Gel-SC gradient structures. d. An illustration of the arm showing the pronator teres muscle attached to the skeleton by two tendons. The illustration is from [36] (Copyright 2003-2004 University of Washington. All rights reserved including all photographs and images. No re-use, re-distribution or commercial use without prior written permission of the authors and the University of Washington.) e. Photograph of the 3D printed gradient gel attached to a skeleton in the same position as the pronator teres.

The artificial tendon-muscle material was attached to the arm of a model skeleton in the position of the pronator teres muscle. The pronator teres muscle functions to rotate the forearm. Figure $4 \mathrm{~d}$. illustrates the anatomical position of the pronator teres muscle and it is attachment to the skeleton by two tendons. Figure 4 e. is a photograph of the artificial tendon when attached to the arm as the 
pronator teres muscle. The example highlights how connection of the artificial tendon to the bone could be made through the harder end regions (in this case Emax $904 \mathrm{Gel-SC}$ ). In future, it is possible to add additional function to the artificial tendon by using a stimuli responsive tough hydrogel in place of the alginate/poly(acrylamide) ICE gel which could cause the artificial tendon to expand and contract in a muscle-like fashion $[14,36]$.

\section{Conclusions}

A new material extrusion based gradient printing system was developed, and its function demonstrated by 3D printing a range of tough hydrogel composites. A spectrum of soft and wet to hard and dry particulate reinforced composites were prepared by altering the ratios of a soft alginate/polyacrylamide based hydrogel to a hard UV-curable ink (Emax $904 \mathrm{Gel}-\mathrm{SC}$ ) in the materials. The printed materials were mechanically characterized in tension and were modeled by composite theory. The 3D printer platform allowed the composites to be fabricated directly into functional structures and this was demonstrated by printing an anatomical model of a tendon. The artificial tendon contained a gradient of composition and mechanical properties between the extremes of the hydrogel and the Emax $904 \mathrm{Gel}-\mathrm{SC}$. The printer itself fills a void within the current suite of 3D printers as the first extrusion based deposition system capable of producing these smooth and continuous gradient structures. An important aspect of the printer function is the use of two mechanically driven syringe pumps in conjunction with new software that allows for independent control over the rate of extrusion from each of the pumps into a static mixer. Each syringe pump can be loaded with a different ink, which is then mixed together in desired ratios by the static mixer. In this project two immiscible inks were printed to fabricate particulate reinforced hydrogels. In the future this printer could also be used to process two miscible inks to form polymer blends or interpenetrating networks. This technique not only opens a pathway towards new functionally graded materials for use in the next generation of anatomical models, but also could ultimately lead 
the way towards the fabrication of multifunctional single material systems that would replace the need for objects requiring a complex assembly of multiple parts with homogeneous properties and discrete functionality $[18,26]$.

\section{Acknowledgements}

The authors would like to thank Professor Paul Calvert for discussions to develop the printing technology and Ali Jeiranikhameneh with support from the Australian National Fabrication Facility for printing the nozzle. Financial support for this work was provided by the University of Wollongong (AlIM for Gold Grant) and the Australian Research Council (DP110101073). The Musculoskeletal Image in Figure 4 is from the University of Washington "Musculoskeletal Atlas: A Musculoskeletal Atlas of the Human Body" by Carol Teitz, M.D. and Dan Graney, Ph.D.

\section{References}

[1] Mironov V, Trusk T, Kasyanov V, Little S, Swaja R, Markwald R. Biofabrication: a 21st century manufacturing paradigm. Biofabrication 2009;1(2):022001.

[2] Yusong P, Qianqian S, Chengling P, Jing W. Prediction of mechanical properties of multilayer gradient hydroxyapatite reinforced poly(vinyl alcohol) gel biomaterial. J Biomed Mater Res A 2013;101B(5):729-35.

[3] Yusong P, Qianqian S, Chengling P, Jing W. Compressive Mechanical Characteristics of Multilayered Gradient Hydroxyapatite Reinforced Poly (Vinyl Alcohol) Gel Biomaterial. J Mater Sci Technol 2013;29(6):551-6.

[4] Zhao X. Multi-scale multi-mechanism design of tough hydrogels: building dissipation into stretchy networks. Soft Matter 2014;10(5):672-87.

[5] Sun J-Y, Zhao X, Illeperuma WRK, Chaudhuri O, Oh KH, Mooney DJ, et al. Highly stretchable and tough hydrogels. Nature 2012;489(7414):133-6. 
[6] Haque MA, Kurokawa T, Gong JP. Super tough double network hydrogels and their application as biomaterials. Polymer 2012;53:(9)1805-22.

[7] Bakarich SE, Pidcock GC, Balding P, Stevens L, Calvert P, in het Panhuis M. Recovery from applied strain in interpenetrating polymer network hydrogels with ionic and covalent cross-links. Soft Matter 2012;8(39):9985-8.

[8] Li J, Illeperuma WRK, Suo Z, Vlassak JJ. Hybrid Hydrogels with Extremely High Stiffness and Toughness. ACS Macro Lett 2014;3(6):520-3.

[9] Agrawal A, Rahbar N, Calvert P. Strong fiber-reinforced hydrogel. Acta Biomater 2013;9(2):5313-

8.

[10] Gong J, Katsuyama Y, Kurokawa T, Osada Y. Double-network hydrogels with extremely high mechanical strength. Adv Mater 2003;15:(14)1155-8.

[11] Mikos AG, Herring SW, Ochareon P, Elisseeff J, Lu HH, Kandel R, et al. Engineering complex tissues. Tissue Eng 2006;12(12):3307-39.

[12] Seidi A, Ramalingam M, Elloumi-Hannachi I, Ostrovidov S, Khademhosseini A. Gradient biomaterials for soft-to-hard interface tissue engineering. Acta Biomater 2011;7(4):1441-51.

[13] Pompe W, Worch H, Epple M, Friess W, Gelinsky M, Greil P, et al. Functionally graded materials for biomedical applications. Mater Sci Eng A 2003;362(1):40-60.

[14] Naficy S, Kawakami S, Sadegholvaad S, Wakisaka M, Spinks GM. Mechanical properties of interpenetrating polymer network hydrogels based on hybrid ionically and covalently crosslinked networks. J Appl Polym Sci 2013;130(4):2504-13.

[15] Jones N. The print revolution Three-dimensional printers are opening up new worlds to research. Nature 2012;487(7405):22-3.

[16] Fan H, Yu Y, Stump A, Reed ST, Baer T, Schunk R, et al. Rapid prototyping of patterned functional nanostructures. Nature 2000;405(6782):56-60.

[17] Zhou MY, Xi JT, Yan JQ. Modeling and processing of functionally graded materials for rapid prototyping. J Mater Process Techno 2004;146(3):396-402. 
[18] Oxman N. Variable property rapid prototyping: inspired by nature, where form is characterized by heterogeneous compositions, the paper presents a novel approach to layered manufacturing entitled variable property rapid prototyping. Virtual Physical Prototyp 2011;6(1):3-31.

[19] Pataky K, Braschler T, Negro A, Renaud P, Lutolf MP, Brugger J. Microdrop printing of hydrogel bioinks into 3D tissue-like geometries. Adv Mater 2012;24(3):391-6.

[20] Billiet T, Vandenhaute M, Schelfhout J, Van Vlierberghe S, Dubruel P. A review of trends and limitations in hydrogel-rapid prototyping for tissue engineering. Biomaterials 2012;33(26):6020-41. [21] Bakarich SE, in het Panhuis M, Beirne S, Wallace GG, Spinks GM. Extrusion printing of ioniccovalent entanglement hydrogels with high toughness. J Mater Chem B 2013;1(38): 4939-46. [22] Hong S, Sycks D, Chan HF, Lin S, Lopez GP, Guilak F, et al. 3D Printing of Highly Stretchable and Tough Hydrogels into Complex, Cellularized Structures. Adv Mater 2015;27(27):4035-40.

[23] Bakarich SE, Balding P, Gorkin III R, Spinks GM, in het Panhuis M. Printed ionic-covalent entanglement hydrogels from carrageenan and an epoxy amine. RSC Adv 2014;4(72):38088-92.

[24] Kirchmajer DM, Gorkin lii R, in het Panhuis M. An overview of the suitability of hydrogel-forming polymers for extrusion-based 3D-printing. J Mater Chem B 2015;3(20):4105-17.

[25] Bakarich SE, Gorkin III R, in het Panhuis M, Spinks GM. Three-Dimensional Printing Fiber Reinforced Hydrogel Composites. ACS Appl Mater Interfaces 2014;6(18):15998-6006.

[26] Mogas-Soldevila L, Duro-Royo J, Oxman N. Water-Based Robotic Fabrication: Large-Scale Additive Manufacturing of Functionally Graded Hydrogel Composites via Multichamber Extrusion. 3D Print Addit Manuf 2014;1(3):141-51.

[27] Shengjie Li, Yongnian Yan, Zhuo Xiong, Chenyang Weng, Renji Zhang, Xiaohong Wang. Gradient Hydrogel Construct Based on an Improved Cell Assembling System. J Bioact Compat Pol 2009;24(1):84-99.

[28] Klein TJ, Rizzi SC, Reichert JC, Georgi N, Malda J, Schuurman W, et al. Strategies for zonal cartilage repair using hydrogels. Macromol Biosci 2009;9(11):1049-58. 
[29] Mire CA, Agrawal A, Wallace GG, Calvert P, in het Panhuis M. Inkjet and extrusion printing of conducting poly(3,4-ethylenedioxythiophene) tracks on and embedded in biopolymer materials. J Mater Chem 2011;21(8):2671-8.

[30] Naficy S, Brown HR, Razal JM, Spinks GM, Whitten PG. Progress Toward Robust Polymer Hydrogels. Aust J Chem 2011;64(8):1007-25.

[31] Hull D. An introduction to composite materials. 1 ed. Cambridge: The Press Syndicate of the University of Cambridge; 1981.

[32] Einstein A. Investigations on the Theory of Brownian Motion, Reprint of the 1st English edition (1926). Dover, New-York; 1956.

[33] Fu S-Y, Feng X-Q, Lauke B, Mai Y-W. Effects of particle size, particle/matrix interface adhesion and particle loading on mechanical properties of particulate-polymer composites. Composites Part B: Engineering 2008;39(6):933-61.

[34] Ahmed S, Jones FR. A review of particulate reinforcement theories for polymer composites. J Mater Sci 1990;25(12):4933-42.

[35] Ishai O, Cohen L. Elastic properties of filled and porous epoxy composites. Int J Mechanical Sci 1967;9(8):539-46.

[36] Teitz, C and Graney,D. "Musculoskeletal Atlas: A Musculoskeletal Atlas of the Human Body" University of Washington, 2003.

[37] Bakarich SE, Gorkin R, in het Panhuis M, Spinks GM. 4D Printing with Mechanically Robust, Thermally Actuating Hydrogels. Macromol Rapid Commun 2015;36(12):1211-7. 